\title{
An Edge Detection Method using a Fuzzy Ensem- ble Approach
}

\author{
Ernesto Moya-Albor, Hiram Ponce, Jorge Brieva* \\ Universidad Panamericana, Campus México, Facultad de Ingeniería, Augusto \\ Rodin 498, México, Ciudad de México, 03920, México \\ emoya@up.edu.mx,hponce@up.edu.mx,jbrieva@up.edu.mx \\ * Corresponding author
}

\begin{abstract}
Edge detection is one of the most important low level steps in image processing. In this work we propose a fuzzy ensemble based method for edge detection including a fuzzy c-means (FCM) approach to define the input membership functions of the fuzzy inference system (FIS). We tested the performance of the method using a public database with ground truth. Also, we compared our proposal with classical and other fuzzy based methods, using F-measure curves and the precision metric. We conducted experiments with different levels of salt \& pepper noise to evaluate the performance of the edge detectors. The metrics illustrate the robustness of the choice of the threshold in the binarization step using this fuzzy ensemble method. In noisy conditions, the proposed method works better than other fuzzy approaches. Comparative results validated that our proposal overcomes traditional techniques.
\end{abstract}

Keywords: edge detection; fuzzy inference system; fuzzy clustering; noise, image processing

\section{Introduction}

Edge detection is one of the most important low level steps in image processing. It is used in several high level analysis as features identification, register and motion estimation [1,2]. From a signal processing point of view, the process consists of the detection of abrupt local changes in intensity, texture or luminosity [3]. The expected final result is a binary image corresponding to the pixels labeled as point contours. This procedure includes two steps: an enhancement of the image contours and a decision step to determine if a pixel is a contour or not on the basis of some local or regional information. This second step remains important and it usually can be done by thresholding [4].

Many techniques have been presented in the literature to solve the edge detection problem. In this paper, we will present the most representatives. Well known traditional techniques use the convolution of derivative-based linear-time invariant (LTI) 
filters with the image, such as: Sobel [5], Roberts [6] and Prewitt [7] detectors. Ulipinar et al. [8] use zero crossings of the Laplacian of Gaussian operator. Canny uses derivative-based filters adding a restriction of gradient direction to eliminate the non-coherent contours [9]. These methods are not robust in noisy images [10] and they remain very dependent of the threshold choice in the binarization step. Also, mathematical morphology approaches are widely used in the segmentation and edge detection problems, since they work very well in the presence of high contrast structures. In the case of noisy images and low contrast structures, some works which attack this problem are presented in the literature. For example, Wang et al. [11] proposed a multi-scale and multi-form method. Jiang et al. [12] developed a mask based on noise filtering. A combination of morphological filtering and the Laplacian operator were proposed by [13], and a histogram based edge detector was carried out by Krishnamurthy et al. [14]. The main weakness of these approaches is the dependency of the choice on the form and scale of the structural element.

Edge detectors using intelligent approaches have been widely used, those using artificial neural networks [15], spatial clustering [16] and adaptive neuro-fuzzy systems [17]. It is well known the merits of these kinds of methods, but the learning step is very dependent of the application. In particular, fuzzy logic has been employed for edge detection $[18,19,20]$. It consists of the definition of a set of rules and membership functions to associate a possibility of true detection to the pixel. In [18], authors proposed an edge detection algorithm based on fuzzy rules to estimate the edge strength, and a threshold is estimated using an optimization algorithm. Setarehdan et al. proposed a fuzzy temporal and multi-scale method applied to a sequence of cardiac images [21]. Moreover, in the presence of noise, the edge detection task becomes more difficult due to the presence of abrupt changes in intensity not corresponding to edges belonging to objects in the image; but fuzzy based techniques can deal with the presence of noise due to its uncertainty property. For instance, Haq et al. [22] proposed a fuzzy logic based edge detection in smooth and noisy clinical images. They employed a $3 \times 3$ mask guided by fuzzy rules set. The mask was formed by the differences of gray level between the center pixel in the mask and each one of the neighbors.

In this work, we propose an edge detection algorithm for gray scale images based on a fuzzy ensemble of both fuzzy inference system and fuzzy c-means clustering. The aim of this method is to exploit global information of an image and use it locally, by a mask technique, to detect edges. The input membership functions are defined by a variable region of uncertainty and an automatic adaptation method to characterize their shapes. Moreover, this method allows to control the mask definition. In addition, an evaluation methodology is proposed and tested for a set of possible thresholds. Different levels of salt \& pepper noise are used to establish the robustness of our proposal.

The remaining article is organized as follows. First, the proposed edge detection based on a fuzzy ensemble is presented. Then, an evaluation methodology and the metrics are described. Comparative results with other well-known methods are carried out, as well as the analysis and discussion of the work. Finally, conclusions and future work are discussed. 


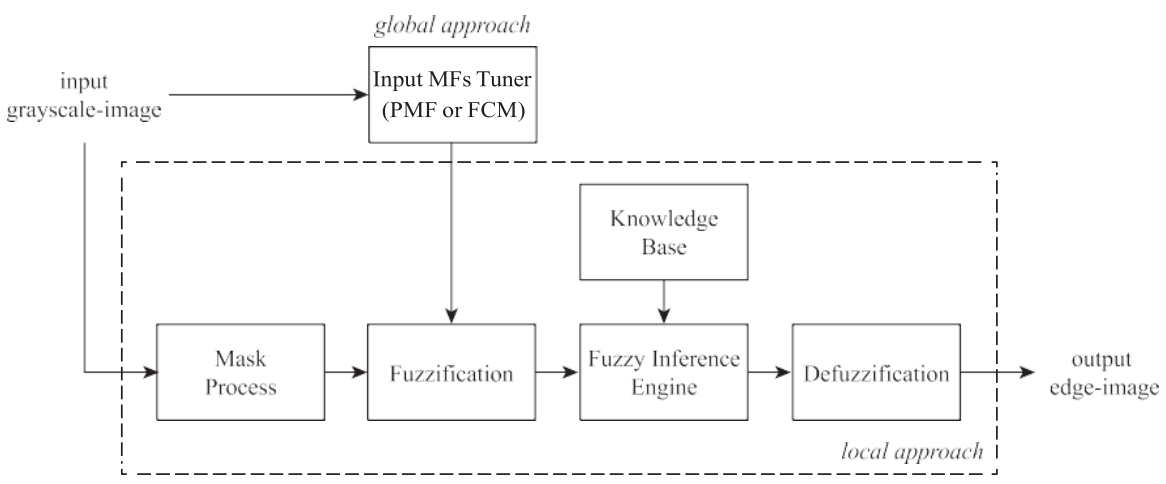

Figure 1

Block diagram of the proposed edge detection algorithm.

\section{Methodology}

The proposed edge detection algorithm for grayscale images is based on a fuzzy ensemble of both fuzzy inference system (FIS) and fuzzy c-means (FCM) clustering. The aim of this method is to exploit global information of an image and use it locally, by a mask technique, to detect edges. As a result, the proposed edge detector can be adapted to images in terms of the intensity values and the present noise.

Figure 1 shows the components of our proposal. As shown, the grayscale image inputs to the input membership functions tuner (global approach) aiming to represent the whole image in terms of the space of difference values by using the FCM method. Then, the input image enters to the mask process (local approach) that uses a $3 \times 3$ window. At each window, the FIS with the tuned input membership functions computes if the central pixel of the window is an edge or not. At the end of the fuzzy-based mask process, an output edge map is obtained. This proposal is described in detail below.

\subsection{Local Approach}

Locally, the proposed edge detection algorithm implements a mask technique consisting on a $3 \times 3$ window, as shown in Figure 2. Considering that $P_{i}$ is the central pixel and $P_{v(\delta)}$ for all $v=1, \ldots, 8$ are the eight neighborhood pixels with radius $\delta$ from $P_{i}$, then the mask computes the difference values between the central pixel and its neighbors with radius $\delta$ as expressed in (1).

$\Delta P_{v(\delta)}=\left|P_{v(\delta)}-P_{i}\right|$

Once the difference values from $P_{i}$ are calculated, the edge detection algorithm employs a fuzzy inference system to classify if $P_{i}$ is an edge pixel or not. 

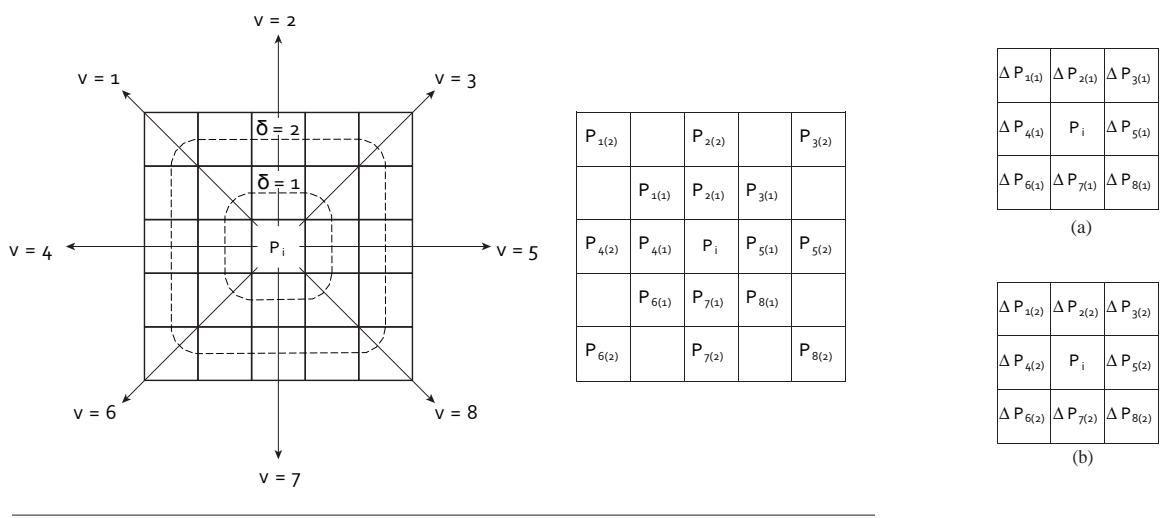

Figure 2

Mask definition in the proposed edge detection algorithm, where $P_{i}$ is the central pixel and $P_{v(\delta)}$ are its neighbors $v$ with radius $\delta$. Example of a $3 \times 3$ window mask: (a) with $\delta=1$ and (b) with $\delta=2$.

\subsubsection{Fuzzification}

The difference values $\Delta P_{v(\delta)}$ for all $v=1, \ldots, 8$ are used as inputs in the fuzzification step of the FIS. Based on literature [22], each input is partitioned into two fuzzy sets: small $\left(F_{v, \text { small }}\right)$ and large $\left(F_{v \text {, large }}\right)$ difference values. Then, both fuzzy sets are represented by membership functions $\mu_{\text {small }}\left(\Delta P_{v(\delta)}\right)$ and $\mu_{\text {large }}\left(\Delta P_{v(\delta)}\right)$.

Actually, the definition of the input membership functions is highly important because they directly influence in the behavior of the FIS [23]. Moreover, these input membership functions are typically tuned in terms of experts in the field [24] or using a priori knowledge of the problem domain [23, 25]. In this proposal, we explore the latter by implementing a global approach that recognizes the whole image and represents it in the input membership functions, as described below.

\subsubsection{Fuzzy Inference Engine and Knowledge Base}

The next step in the FIS is the fuzzy inference engine. It receives the input membership values computed in the step before and performs an inference operation in the fuzzy space in order to obtain a fuzzy consequence value $p_{s}$ that describes the belonging of the central pixel $P_{i}$ to be an edge pixel or not. If the inference operation is described as a fuzzy rule, the $s$-th fuzzy rule $R_{s}$ can be expressed as (2); where, $\wedge$-operator represents the T-norm in the fuzzy inference. In this work, the min-operator is selected as the T-norm. To this end, the set of fuzzy rules is based on literature [22] and in additional information extracted from a prior analysis to different configurations of masks detecting edges as summarized in Table 1.

$R_{S}:$ if $\Lambda_{v=1}^{8} \Delta P_{v(\delta)} \in F_{v}$, then $p_{s} \in P_{i}$ 
Table 1

Summary of fuzzy rules detecting an edge pixel

\begin{tabular}{|c|c|c|c|c|c|c|c|c|c|}
\hline rule & $\Delta P_{1(\mathrm{\delta})}$ & $\Delta P_{2(\delta)}$ & $\Delta P_{3(\mathrm{\delta})}$ & $\Delta P_{4(\mathrm{\delta})}$ & $\Delta P_{5(\mathrm{\delta})}$ & $\Delta P_{6(\mathrm{\delta})}$ & $\Delta P_{7(\mathrm{\delta})}$ & $\Delta P_{8(\mathrm{\delta})}$ & $P_{i}$ \\
\hline 1 & large & large & - & - & - & - & - & small & edge \\
\hline 2 & large & - & - & large & - & - & - & small & edge \\
\hline 3 & - & large & large & - & - & - & - & small & edge \\
\hline 4 & - & - & - & large & - & large & - & small & edge \\
\hline 5 & large & large & - & - & - & - & small & - & edge \\
\hline 6 & large & - & - & large & - & - & small & - & edge \\
\hline 7 & - & large & large & - & - & - & small & - & edge \\
\hline 8 & - & - & - & large & - & large & small & - & edge \\
\hline 9 & large & large & - & - & small & - & - & - & edge \\
\hline 10 & large & - & - & large & small & - & - & - & edge \\
\hline 11 & - & large & large & - & small & - & - & - & edge \\
\hline 12 & - & - & - & large & small & large & - & - & edge \\
\hline 13 & - & - & small & - & - & large & large & - & edge \\
\hline 14 & - & - & small & - & - & - & large & large & edge \\
\hline 15 & - & small & - & - & - & large & large & - & edge \\
\hline 16 & - & - & large & - & large & small & - & - & edge \\
\hline 17 & - & - & - & - & large & small & - & large & edge \\
\hline 18 & - & - & large & - & large & - & small & - & edge \\
\hline 19 & - & small & - & - & - & - & large & large & edge \\
\hline 20 & - & - & - & - & large & - & small & large & edge \\
\hline 21 & - & - & - & - & small & large & large & - & edge \\
\hline 22 & - & - & large & small & large & - & - & - & edge \\
\hline 23 & - & - & - & - & small & - & large & large & edge \\
\hline 24 & - & - & - & small & large & - & - & large & edge \\
\hline & & & & & & & & & - \\
\hline
\end{tabular}

\subsubsection{Defuzzification}

The last step of the FIS considers the defuzzification process. It calculates the crisp output value $P_{i}$ representing if the central pixel is an edge pixel or not. In this work, we use the center of gravity approach [26] expressed as (3); where $\mu_{s}\left(p_{t}\right)$ represents the membership value of $p_{t}$, an intensity value in the space of edges.

$$
P_{i}=\frac{\sum_{t} \mu_{s}\left(p_{t}\right) \cdot p_{t}}{\sum_{t} \mu_{s}\left(p_{t}\right)}
$$

At last, the output of the proposed edge detector is partitioned into two fuzzy sets: non-edge $\left(P_{n e}\right)$ and edge $\left(P_{e}\right)$, represented as Gaussian membership functions with means 10 and 245 and with standard deviation 3.5.

\subsection{Global Approach}

As already said, the global approach of the proposed edge detection algorithm corresponds to define the input membership functions of the FIS aiming to represent the whole image in terms of the space of difference values. This enables to the algorithm getting an overall perspective of the image and the frequency of difference values across it. To accomplish it, we propose two methods: one based on the probability distribution of difference values in the image, and the other based on a fuzzy clustering of the difference values in the image. 


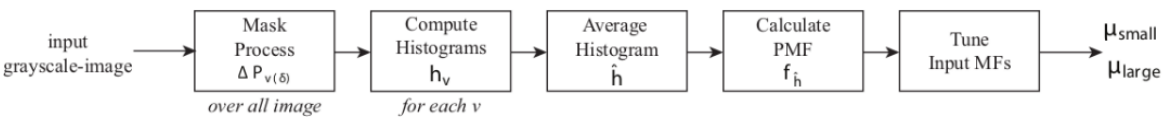

Figure 3

Block diagram of the global approach based on the probability mass function of the difference values

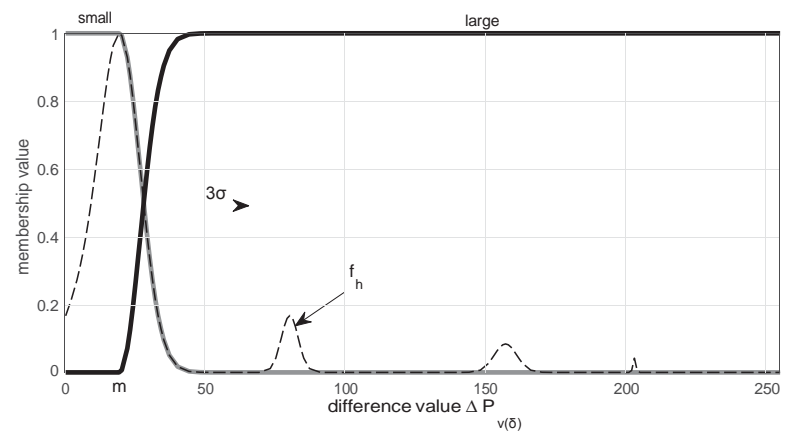

Figure 4

Input membership functions derived from the probability distribution of difference values in the image

\subsubsection{Method Based on Probability Distribution}

The first method consists on determine the probability mass function (PMF) of the difference values in the whole image. Figure 3 shows the block diagram of this method.

At first, the $3 \times 3$ mask is used to calculate the difference values $\Delta P_{v(\delta)}, v=1, \ldots, 8$, with radius $\delta$ from the central pixel $P_{i}$. Then, a histogram $h_{v}$ is computed for each direction $v$ in the neighborhood. The histogram contains the number of bins as the number of integer values has the input $\Delta P_{v(\delta)}$ in the universe of discourse in the FIS. For instance, if the universe of discourse of the difference values ranges from 0 to 255 , then the histogram will have 256 bins. Notice that all the histograms will have the same number of bins since the difference values in any direction are computed over the same grayscale values. To this end, we propose to use the number of gray levels in the image as the universe of discourse, and also in the number of bins in histograms. Secondly, an average histogram $\hat{h}$ is computed using all the histograms $h_{v}$ from $v=1, \ldots, 8$. Then, the probability mass function $f_{\hat{h}}\left(\Delta P_{v(\delta)}\right)$ of the average of difference values in every direction is expressed as (4).

$$
f_{\widehat{h}}\left(\Delta P_{v(\delta)}\right)=\frac{\widehat{h}}{\max (\widehat{h})}
$$

Considering that homogeneous regions dominate in the image, then the probability mass function will shape at least one peak, i.e. the largest, in the left side representing the most frequent difference values in the image. In fact, this peak represents 
small difference values, and it is close to the mean value $m$ of $f_{\hat{h}}$. Thus, the left side of the peak in $f_{\hat{h}}$ is proposed to be used as part of the input membership function small, as denoted in (5); where, $\sigma$ represents the standard deviation of $f_{\hat{h}}$, and $\Delta P_{\max }=\arg \max _{\Delta P_{v(\delta)}}\left(f_{\hat{h}}\right)$. To this end, the input membership function large is the complement of the small one, as expressed in (6). Figure 4 shows the input membership functions defined by this method.

$$
\mu_{\text {small }}\left(\Delta P_{v(\delta)}\right)=\left\{\begin{array}{cc}
1 & \Delta P_{v(\delta)}<\Delta P_{\text {max }} \\
f_{\widehat{h}}\left(\Delta P_{v(\delta)}\right) & \Delta P_{\text {max }} \leq \Delta P_{v(\delta)}<\Delta P_{\text {max }}+3 \sigma \\
0 & \Delta P_{v(\delta)} \geq \Delta P_{\text {max }}+3 \sigma
\end{array}\right.
$$

$\mu_{\text {large }}\left(\Delta P_{v(\delta)}\right)=1-\mu_{\text {small }}\left(\Delta P_{v(\delta)}\right)$

\subsubsection{Method Based on Fuzzy C-Means}

The second method consists on divide the difference values in the whole image using fuzzy c-means clustering technique. Figure 5 shows the block diagram of this method. Fuzzy c-means is a clustering method that groups data using a similarity metric, but also it computes the degree of membership of each data point with respect to each class [27].

In this proposal, FCM clusters the bins (i.e. the difference values $\Delta P_{v(\delta)}$ ) of the average histogram $\hat{h}$, or alternatively of the probability mass function $f_{\hat{h}}$, by minimizing the modified objective function in (7); where, $p_{i}$ is the $i$ th difference value in the set $\Omega=\mathrm{U}_{v} \Delta P_{v(\delta)}$ of all difference values, $c_{j}$ is the center of the $j$ th cluster, $d$ is the number of difference values in $\Omega, k$ is the number of clusters, $\mu_{i j}$ is the degree of membership of $p_{i}$ in the $j$ th cluster, and $s>1$ is the exponent of the fuzzy partition matrix for controlling the degree of fuzzy overlap (in this work, we use $s=2$ ). It is remarkable to say that FCM constrains the degree of membership for a given data point $p_{i}$ such that their membership values sum one.

$$
J_{m}=\sum_{i=1}^{d} \sum_{j=1}^{k} \mu_{i j}^{2}\left\|p_{i}-c_{i}\right\|^{2}, \sum_{j=1}^{k} \mu_{i j}=1
$$

Using the above method, all the difference values $p_{i} \in \Omega$ can be clustered in two groups (i.e. $k=2$ ): small and large values. In that sense, we propose to define the input membership functions of the FIS to be the membership values $\mu_{i j}$ obtained so far from the FCM algorithm. Thus, the input membership function small is proposed

to be as (8); where, $\Delta P_{\max }=\arg \max _{\Delta P_{v(\delta)}}\left(\mu_{i 1}\right)$ and $\Delta P_{\min }=\arg \min _{\Delta P_{v(\delta)}}\left(\mu_{i 1}\right)$ such that $\Delta P_{\min }>\Delta P_{\max }$ if $c_{1}<c_{2}$ (i.e. $c_{1}$ represents the left-class). Again, the input membership function large is proposed to be as (6). To this end, a look-up table or a hash function should be defined in order to use the $\mu_{i j}$ values as part of the input 


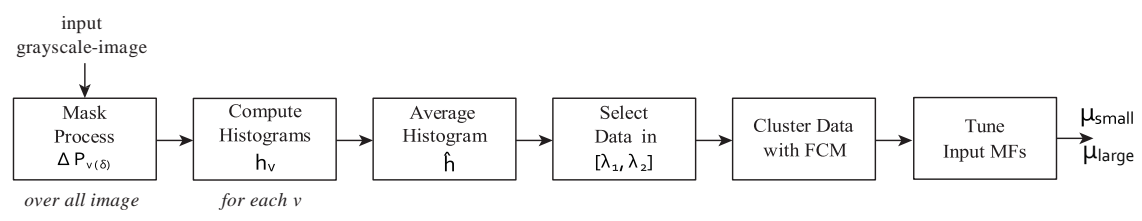

Figure 5

Block diagram of the global approach based on the FCM clustering of the difference values.

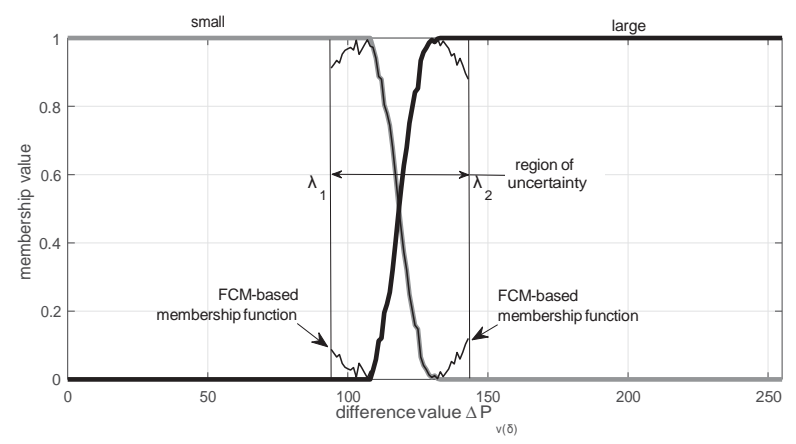

Figure 6

Input membership functions derived from the FCM clustering of difference values in the image

membership functions. Figure 6 shows the input membership functions defined by this method.

$$
\mu_{\text {small }}\left(\Delta P_{v(\delta)}\right)=\left\{\begin{array}{cc}
1 & \Delta P_{v(\delta)}<\Delta P_{\text {max }} \\
\mu_{i 1}\left(\Delta P_{v(\delta)}\right) & \Delta P_{\text {max }} \leq \Delta P_{v(\delta)}<\Delta P_{\text {min }} \\
0 & \Delta P_{v(\delta)} \geq \Delta P_{\text {min }}
\end{array}\right.
$$

It is important to notice that clustering every difference value $p_{i}$ in the set $\Omega$ of all difference values in the image, does not represent the uncertainty region between small and large difference values in a suitable form. Then, the FCM method should be applied only in a region of interest inside the average histogram $\hat{h}$, or alternatively inside the probability mass function $f_{\hat{h}}$. If we denote the region of interest to be the interval $\Lambda=\left[\lambda_{1}, \lambda_{2}\right] \subset \Omega$, then we propose to use the FCM method only with data points in the subset $\Lambda$ such that $p_{i} \in \Lambda$, as denoted in (9). Finally, the overall FCM-based algorithm as the global approach of the edge detector is summarized in Algorithm 1.

$$
J_{m}=\sum_{i} \sum_{j=1}^{k=2} \mu_{i j}^{2}\left\|p_{i}-c_{i}\right\|^{2} \quad, \quad \forall p_{i} \in\left[\lambda_{1}, \lambda_{2}\right]
$$




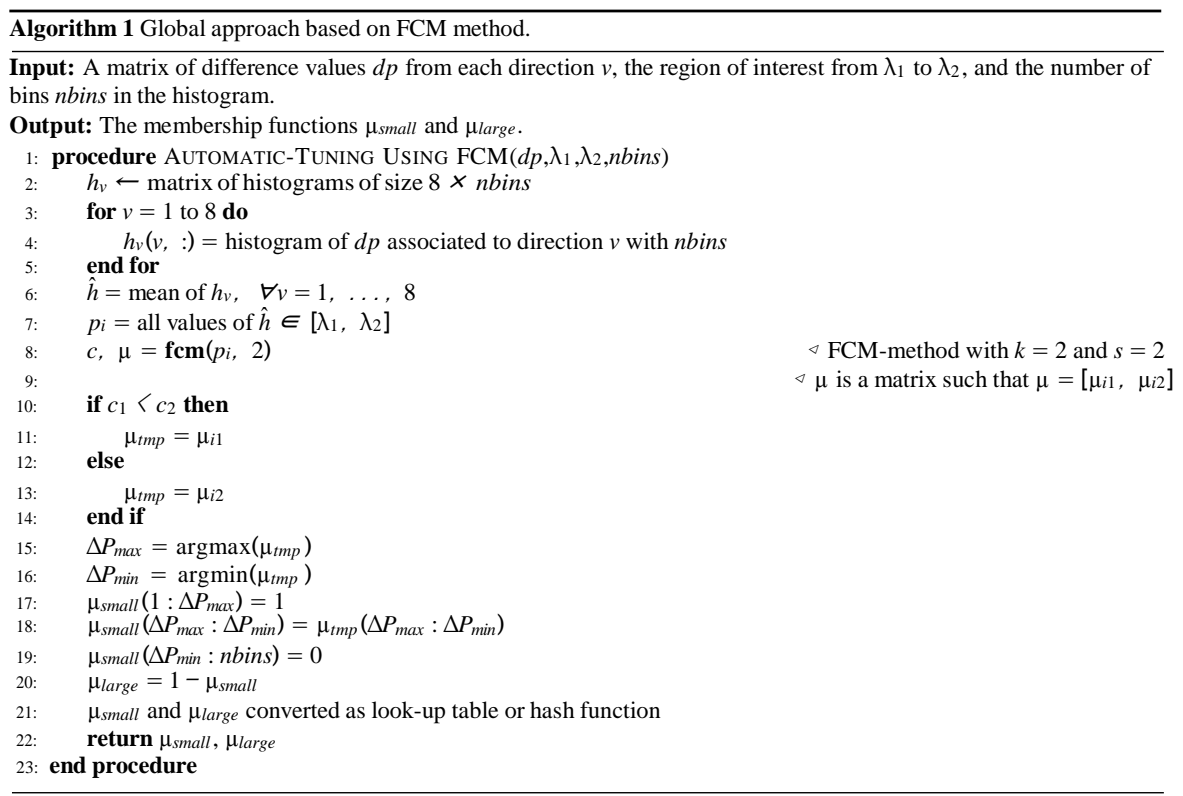

\section{Results and Discussion}

In this section, we evaluate the performance of our proposed fuzzy ensemble based edge detection method using a public database and compare it with well-known classical methods (Sobel, Prewitt, LoG, Roberts, Canny) and the fuzzy based method proposed by [22].

\subsection{Materials}

To evaluate our proposal, we used the "Berkeley Segmentation Dataset and Benchmark BSD300" [28] available from [29], which is formed by natural images manually segmented by some subjects (between 5 and 10). The ground truth (GT) is formed by the superposition of weighted manually segmented annotations. In Figure 7 we show the selected images, and its corresponding ID in the database, in which we applied the edge detection methods.

\subsection{Evaluation Method for Edge Detection}

We conducted several experiments to test the performance of our method on natural and noisy images. The evaluation method consisted on the following steps: (i) application of the edge detection algorithm to an image, (ii) normalization of the edges map (for these experiments, between 0 to 255), (iii) binarization of the edge map by a given set of $N$ thresholds, and (iv) comparison of the $N$ binary images against to the ground truth. In the last step, we carried out a matching edges process as in [29] between each binary image and the ground truth using the libraries for graph 

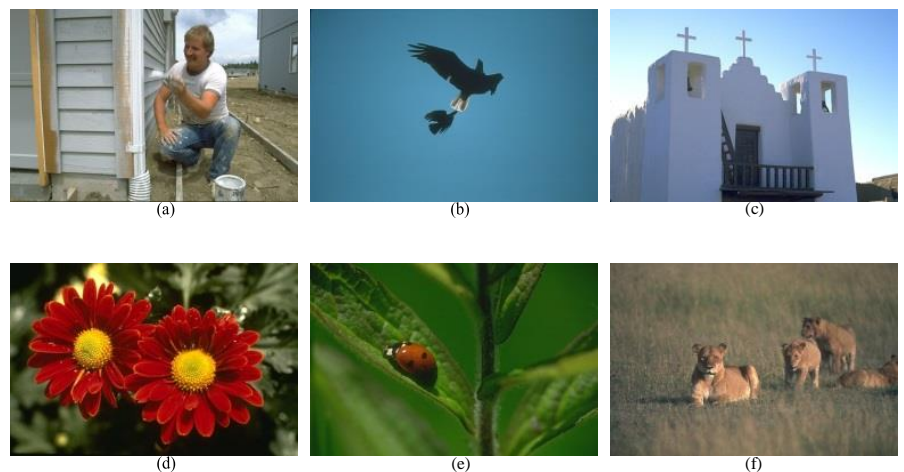

Figure 7

Selected images for experimentation extracted from the BSD300 dataset. IDs: (a) 23080, (b) 135069, (c) 24063, (d) 124084, (e) 35058 and (f) 105053.

assignment problem of Andrew Goldberg's CSA package [30]. Then, we applied different metrics (precision, recall and F-measure) and we obtained F-measure vs threshold curves for each edge detection method.

For the boundary detection problem, some metrics were used to measure the performance of the method tested, the most used are precision $(P)$, recall $(R)$ and F- measure $(F)$. Precision is defined as the ratio of edges that are true positives rather than false positives, or the probability that the edge detector is valid; whereas re-call is the ratio of edges that are true positives detected rather than missed, or the probability that the reference data was detected [31]. Another metric that allows to measure the effectiveness of the algorithm equally in terms of precision and recall is F-measure, that captures the trade-off between precision and recall, as the weighted harmonic mean.

One important issue in the parametric methods for the image processing is the sensitivity analysis of the parameters. To this end, we tried different instances of our proposed method in order to find a suitable set of parameters. These instances are proposed in terms of mask definition $(\delta)$ and the region of uncertainty ( $\left[\lambda_{1}\right.$, $\left.\lambda_{2}\right]$ ). Table 2 shows these variations $\left(F_{1}-F_{7}\right)$ as well as the parameters used in classical methods of edge detection.

\subsubsection{Computation of Suitable Parameters}

Using the evaluation method described above and given a set of thresholds (between 10 and 250 with steps of 10), we show F-measure vs threshold curves for the selected images in Figure 8 for selected images. From these results, we may observe that our proposals $\left(F_{1}-F_{7}\right)$ are competitive with the classical methods found in the literature $(\mathrm{Sb}, \mathrm{Pr}, \mathrm{LoG}, \mathrm{Rb}$ and $\mathrm{Cn}$ ) and comparable with the method of [22] $(\mathrm{Fz})$.

By inspection in Figure 8, it can be seen clearly two different clusters: traditional and fuzzy based methods. The classical ones are less robust with respect to thresh- 
Table 2

Labels and parameters for edge detection methods

\begin{tabular}{|c|c|c|}
\hline Label & Method & Parameters \\
\hline$F z$ & Fuzzy based Haq et al. [22] & $\delta=1$ and $\left[\lambda_{1}=25, \lambda_{2}=75\right]$ \\
\hline$S b$ & Sobel & $3 \times 3$ kernel \\
\hline$P r$ & Prewitt & $3 \times 3$ kernel \\
\hline$L o G$ & LoG & $3 \times 3$ kernel \\
\hline$R b$ & Roberts & $3 \times 3$ kernel \\
\hline $\mathrm{Cn}$ & Canny & $3 \times 3$ kernel \\
\hline$F_{1}$ & Our proposal 1 & $\begin{array}{c}\delta=1 \text { and }\left[\lambda_{1}, \lambda_{2}\right] \text { were estimated automatically } \\
\text { using PMF and FCM based methods }\end{array}$ \\
\hline$F_{2}$ & Our proposal 2 & $\begin{array}{c}\delta=2 \text { and }\left[\lambda_{1}, \lambda_{2}\right] \text { were estimated automatically } \\
\text { using PMF and FCM based methods }\end{array}$ \\
\hline$F_{3}$ & Our proposal 3 & $\delta=1$ and $\left[\lambda_{1}=10, \lambda_{2}=60\right]$ \\
\hline$F_{4}$ & Our proposal 4 & $\delta=2$, and $\left[\lambda_{1}=10, \lambda_{2}=60\right]$ \\
\hline$F_{5}$ & Our proposal 5 & $\begin{array}{c}\delta=1 \text { and }\left[\lambda_{1}, \lambda_{2}\right] \text { were estimated } \\
\text { by PMF based method }\end{array}$ \\
\hline$F_{6}$ & Our proposal 6 & $\begin{array}{c}\delta=2 \text { and }\left[\lambda_{1}, \lambda_{2}\right] \text { were estimated } \\
\text { by PMF based method }\end{array}$ \\
\hline$F_{7}$ & Our proposal 7 & $\begin{array}{c}\delta=1 \text { and }\left[\lambda_{1}=20, \lambda_{2}=50\right] \\
\text { using FCM based method }\end{array}$ \\
\hline
\end{tabular}

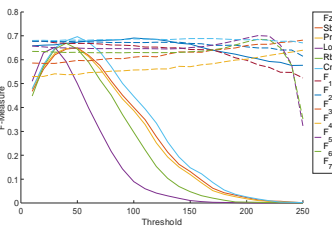

(a)

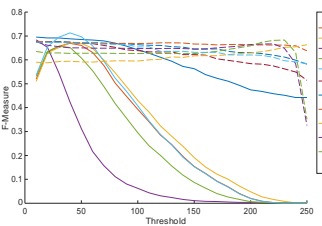

(d)
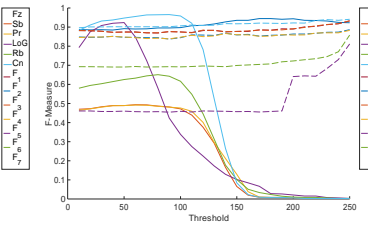

(b)
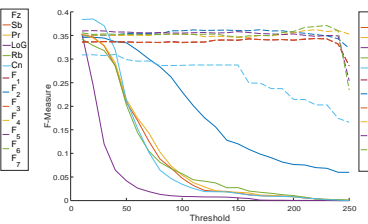

(e)

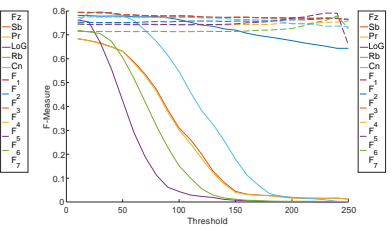

(c)

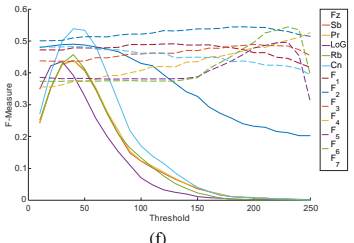

(f)

Figure 8

F-measure vs threshold curves for the selected images. IDs: (a) 23080, (b) 135069, (c) 24063, (d) 124084 , (e) 35058 and (f) 105053.

old in contrast to the fuzzy based methods described in this work. Quantitatively, the robustness dependency can be measured by computing the F-measure standard deviation for all methods over selected images, as shown in Figure 9. An important evidence from the latter is that the fuzzy methods have standard deviation values close to 0.1 , observing that the instances of our proposal are less than this value. Thus, fuzzy based methods are less dependent to threshold. Therefore, we fixed the threshold at the middle range of edges maps (128) for the following experiments, allowing to define an automatic binarization method with a fixed threshold.

In order to determine the suitable variation of our method, we computed the Fmeasure and precision at the fixed threshold for our proposals $\left(F_{1}-F_{7}\right)$ as summarized in Tables 3 and 4, respectively. In Table 3, we may observe that the best set of parameters corresponds to the tests $F_{2}, F_{3}$ and $F_{7}$, and the precision measure shown 


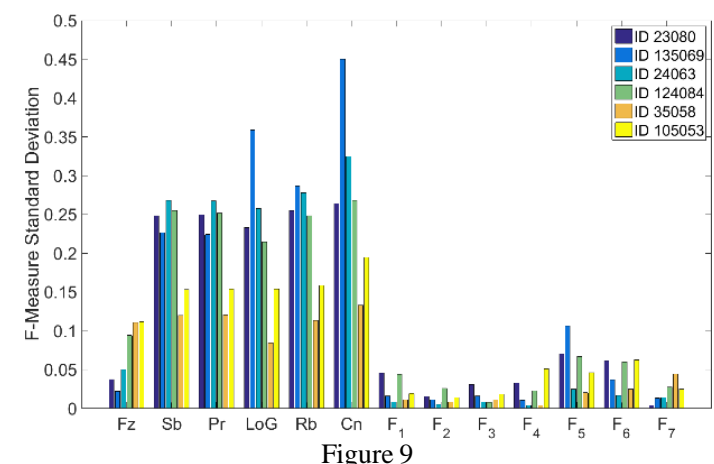

F-measure standard deviation for all methods over selected images.

Table 3

F-measure for fixed threshold

\begin{tabular}{|c|c|c|c|c|c|c|c|}
\hline ID & $F_{1}$ & $F_{2}$ & $F_{3}$ & $F_{4}$ & $F_{5}$ & $F_{6}$ & $F_{7}$ \\
\hline 23080 & 0.653 & 0.672 & 0.623 & 0.561 & 0.645 & 0.628 & $\mathbf{0 . 6 8 4}$ \\
\hline 135069 & 0.884 & 0.857 & 0.884 & 0.854 & 0.456 & 0.691 & $\mathbf{0 . 9 0 8}$ \\
\hline 24063 & 0.773 & 0.755 & $\mathbf{0 . 7 7 3}$ & 0.742 & 0.742 & 0.713 & 0.771 \\
\hline 124084 & 0.633 & 0.655 & $\mathbf{0 . 6 7 1}$ & 0.611 & 0.648 & 0.627 & 0.636 \\
\hline 35058 & 0.341 & $\mathbf{0 . 3 6 1}$ & 0.340 & 0.348 & 0.355 & 0.353 & 0.288 \\
\hline 105053 & 0.490 & $\mathbf{0 . 5 3 2}$ & 0.462 & 0.418 & 0.381 & 0.376 & 0.448 \\
\hline
\end{tabular}

Table 4

Precision measure for fixed threshold

\begin{tabular}{|c|c|c|c|c|c|c|c|}
\hline ID & $F_{1}$ & $F_{2}$ & $F_{3}$ & $F_{4}$ & $F_{5}$ & $F_{6}$ & $F_{7}$ \\
\hline 23080 & $\mathbf{0 . 7 3 0}$ & 0.692 & 0.478 & 0.402 & 0.511 & 0.490 & 0.618 \\
\hline 135069 & 0.803 & 0.752 & 0.804 & 0.748 & 0.296 & 0.528 & $\mathbf{0 . 8 5 2}$ \\
\hline 24063 & 0.746 & 0.699 & 0.747 & 0.642 & 0.620 & 0.577 & $\mathbf{0 . 8 2 0}$ \\
\hline 124084 & 0.640 & $\mathbf{0 . 6 5 7}$ & 0.569 & 0.466 & 0.524 & 0.491 & 0.628 \\
\hline 35058 & 0.343 & 0.326 & 0.342 & 0.269 & 0.246 & 0.241 & $\mathbf{0 . 5 4 1}$ \\
\hline 105053 & 0.414 & 0.439 & 0.334 & 0.272 & 0.241 & 0.234 & $\mathbf{0 . 4 6 9}$ \\
\hline
\end{tabular}

in Table 4 reports that the best results correspond to the test $F_{7}$. According to previous results, we selected $F_{7}$ to be the method with suitable parameters $(\delta=1$ and $\left.\left[\lambda_{1}=20, \lambda_{2}=50\right]\right)$. As an example of the results obtained, in Figure 10 we report the edge detection results using our $F_{7}$ method without binarization. For contrasting

with the other methods, Figure 11 shows an image example (ID image 24063) of the edge detection results for the fixed threshold. It can be seen that the best edge detection corresponds to $\mathrm{Fz}, \mathrm{Cn}$ and $F_{7}$ methods. However, $\mathrm{Fz}$ and $F_{7}$ approaches detect more details on the image. Moreover, $F_{7}$ preserves more edges than $F z$.

\subsubsection{Experiments on Noisy Images}

In order to prove the robustness of our method in presence of noise, we tested it using images corrupted with 35,30 and $24 \mathrm{~dB}$ of peak signal to noise ratio (PSNR) levels of salt \& pepper noise. As concluded previously, the following experiments were carried out using the set of parameters corresponding to $F_{7}$ instance.

The same evaluation method was used to compare the performance of our proposed 

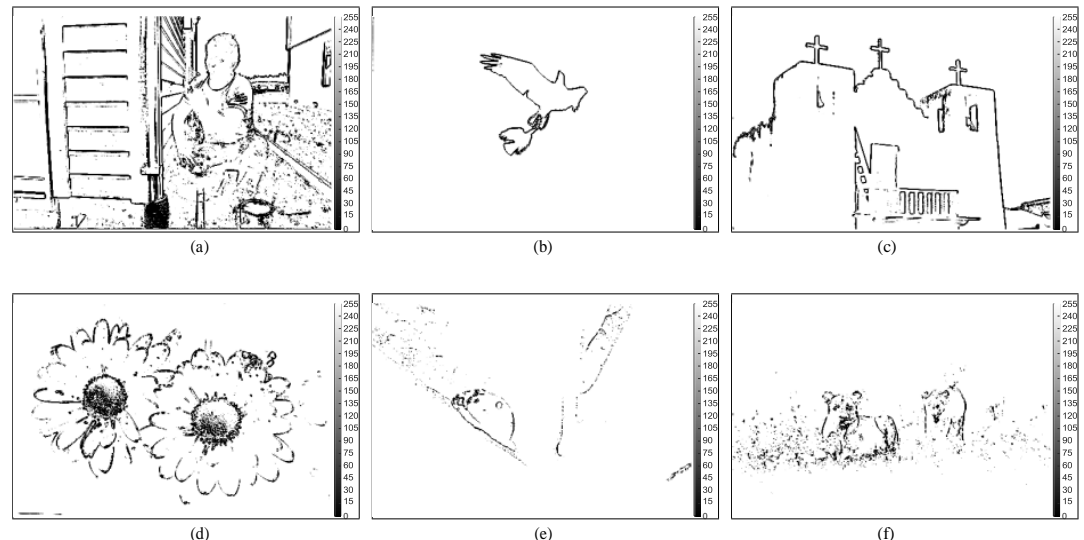

Figure 10

The edge maps resulting from $F_{7}$ method for images: (a) 23080, (b) 135069, (c) 24063, (d) 124084, (e) 35058 and (f) 105053 .
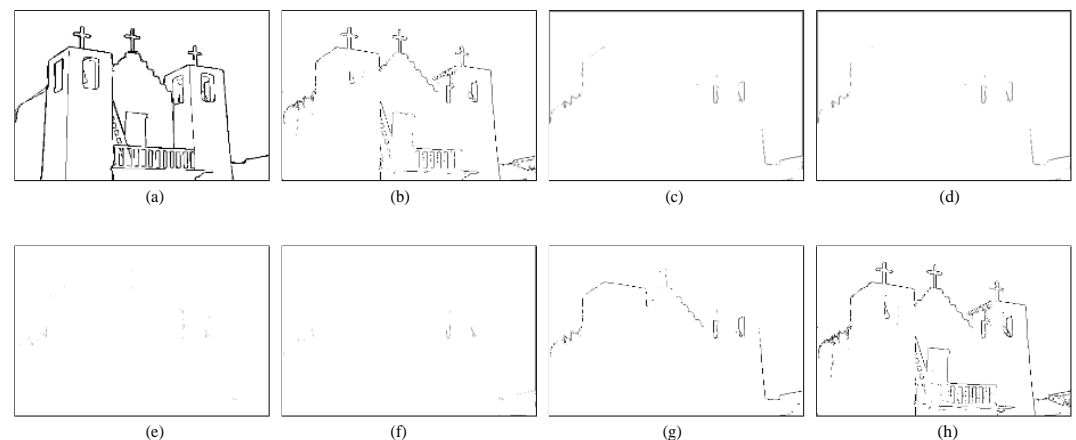

Figure 11

The edge maps resulting using a fixed threshold for all methods (ID Image 24063). Methods: (a) GT, (b) $F z$, (c) $S b$, (d) $P r$, (e) $L o G$, (f) $R b$, (g) $C n$ and (h) $F_{7}$.

fuzzy ensemble based edge detection approach. Figure 12 shows the F-measure vs threshold curves for each edge detection method. Columns represent the noise levels $(35,30$ and $24 \mathrm{~dB}$ of PSRN) and rows correspond to the ID images. Analyzing them, we observed that traditional methods are less robust against to noise levels than fuzzy based methods. For instance, in Figure 12 (g-i), we see that in the best traditional method (Canny) the F-measure decreases $25 \%$ in the different noise levels values $(35,30,24 \mathrm{~dB}$ of PSNR). In contrast, the fuzzy based methods remain almost constant independently to noise level (the F-measure decrease only $1.2 \%$ approximately). We may appreciate that the $F_{7}$ method is more robust with respect to threshold variation than $F z$ method. The same behavior can be seen in all the images.

Figures 13 and 14 report the binarization edge detection results using all the methods (rows) at different noise levels (columns) for the image 24063. In general, we see degradation of edges detection and addition of false edges because of the increasing 


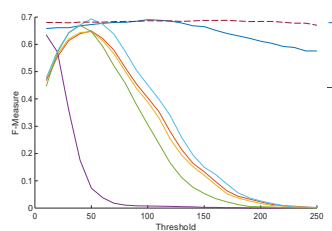

(a)

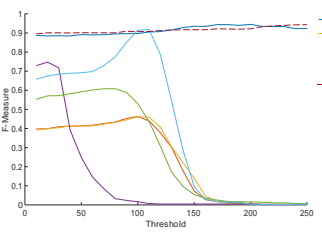

(d)

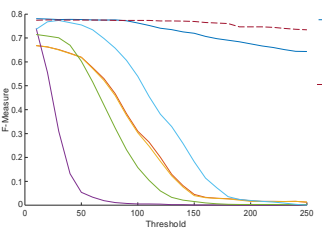

(g)

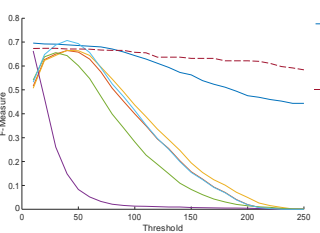

(j)

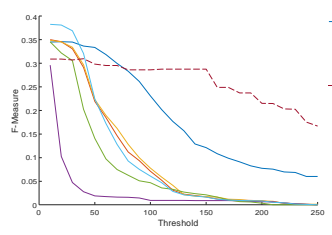

(m)

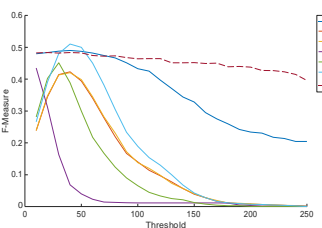

(p)

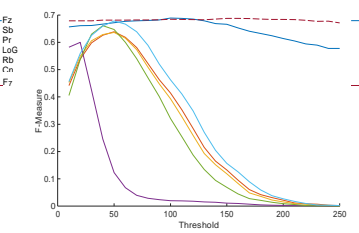

(b)

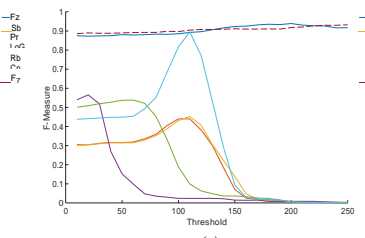

(e)

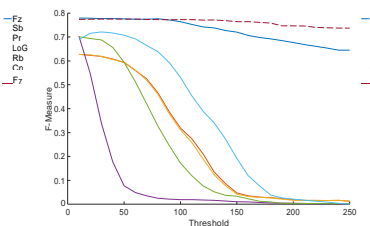

(h)

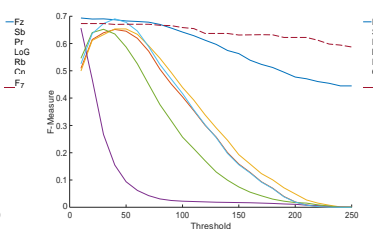

(k)

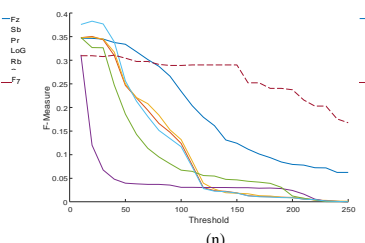

(n)

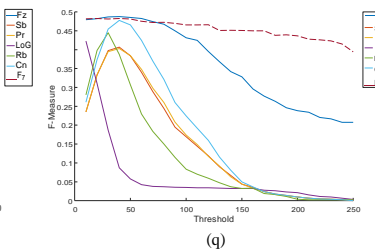

(q)

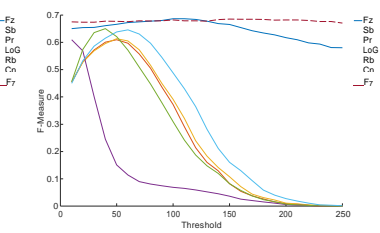

(c)
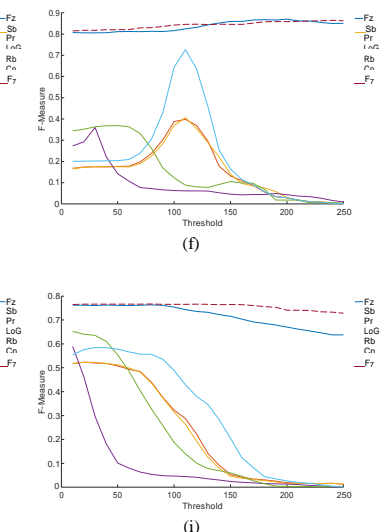

(i)

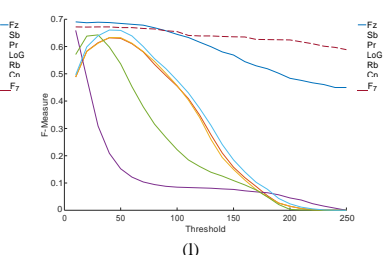

(1)

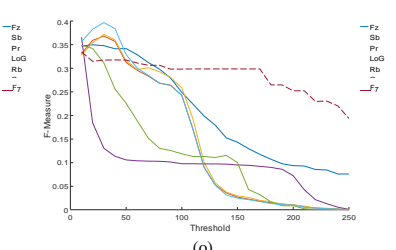

(o)

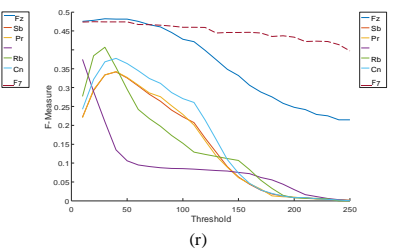

Figure 12

F-measure vs threshold curves for different levels of noise. ID/PSNR: (a) 23080/35dB, (b) 23080/30dB, (c) $23080 / 24 \mathrm{~dB}$, (d) $135069 / 35 \mathrm{~dB}$, (e) $135069 / 30 \mathrm{~dB}$, (f) $135069 / 24 \mathrm{~dB}$, (g) $24063 / 35 \mathrm{~dB}$, (h) $24063 / 30 \mathrm{~dB}$, (i) $24063 / 24 \mathrm{~dB}$, (j) $124084 / 35 \mathrm{~dB}$, (k) $124084 / 30 \mathrm{~dB}$, (l) $124084 / 24 \mathrm{~dB}$, (m) $35058 / 35 \mathrm{~dB}$, (n) $35058 / 30 \mathrm{~dB}$, (o) $35058 / 24 \mathrm{~dB}$, (p) $105053 / 35 \mathrm{~dB}$, (q) $105053 / 30 \mathrm{~dB}$ and (r) $105053 / 24 \mathrm{~dB}$. 

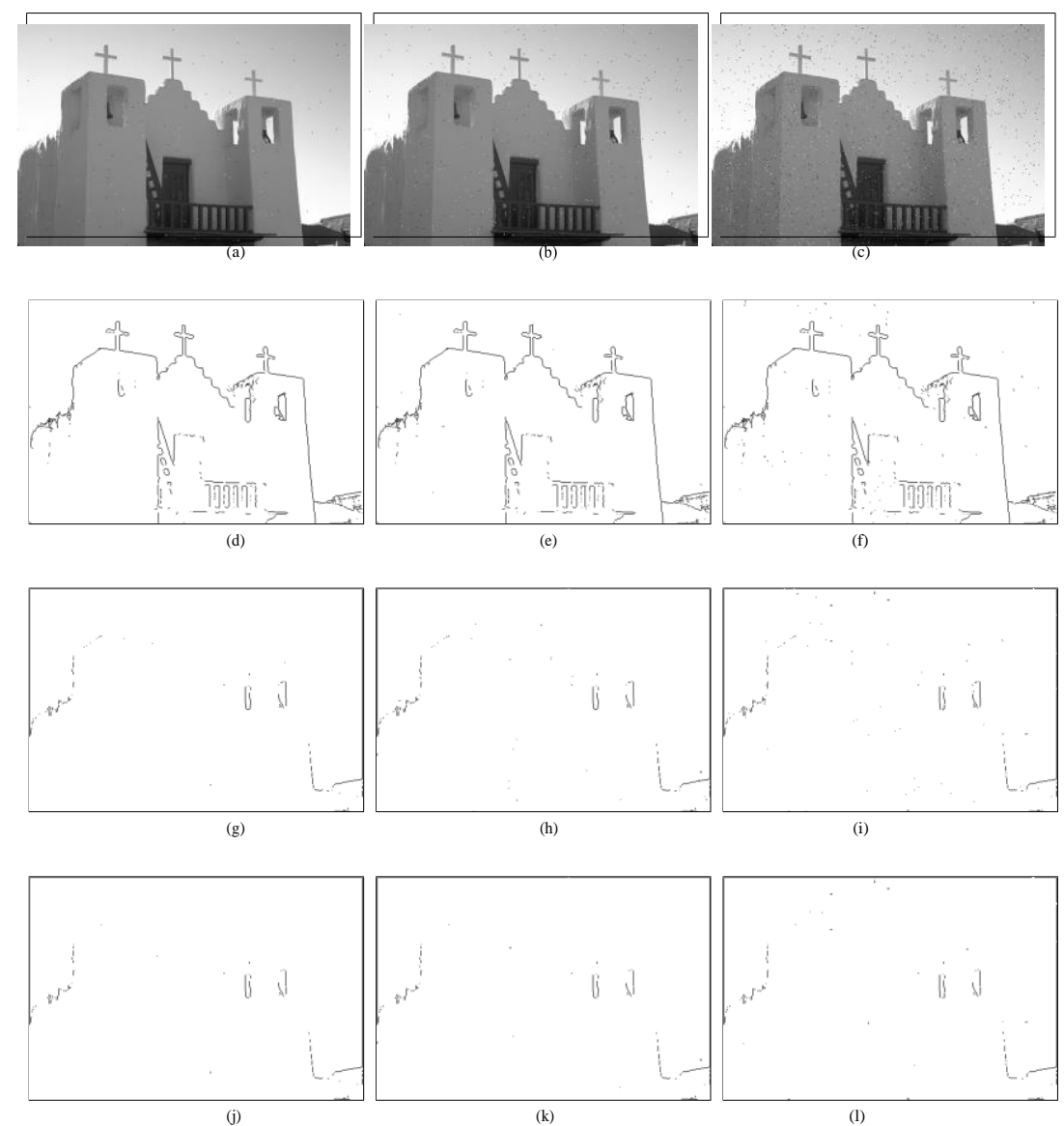

Figure 13

Binarization results in noisy image 24063 using a fixed threshold. (a) PSNR 35dB, (b) PSNR 30dB and (c) PSNR 24dB. Method/PSNR: (d) $F z / 35 \mathrm{~dB}$, (e) $F z / 30 \mathrm{~dB}$, (f) $F z / 24 \mathrm{~dB}$, (g) $S b / 35 \mathrm{~dB}$, (h) $S b / 30 \mathrm{~dB}$, (i) $S b / 24 \mathrm{~dB}$, (j) $P r / 35 \mathrm{~dB}$, (k) $P r / 30 \mathrm{~dB}$ and (1) $P r / 24 \mathrm{~dB}$.

of salt \& pepper noise level. In addition, $\mathrm{Fz}, \mathrm{Cn}$ and $F_{7}$ methods give the best responses independently to the noise level. At first, we can see that fuzzy based methods detect less noise than Canny method. In comparison to $F z, F_{7}$ method preserves more edge details.

In Figure 15, we show all the edge detection images for the highest noise level (24 dB) using the $F_{7}$ method. In Figures 15(b and c), it can be seen well defined and proper edges maps corresponding to images with homogenous backgrounds, as noted in curves Figures 12(f and i). For the high contrast images, Figures 15(a and d), the edges remain well detected, as observed in Figures 12(c and 1). However, $F_{7}$ method is not robust for low contrast images as shown in Figures 15(e and f). Nevertheless, the other methods used in this work also have the same problem for low contrast images as depicted in Figure 12(o and r). 

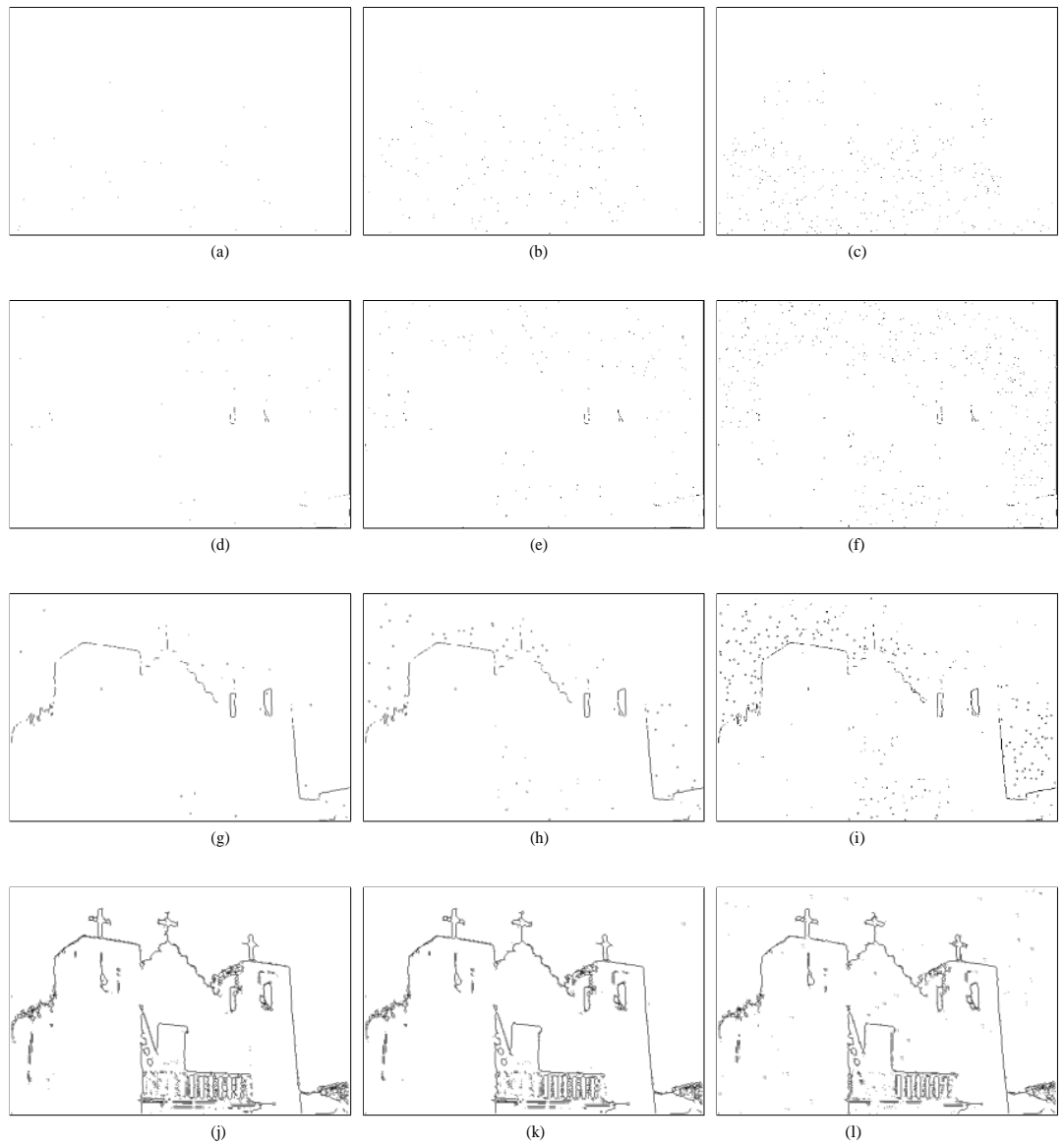

Figure 14

Cont. Binarization results in noisy images using a fixed threshold. Method/PSNR: (a) $L o G / 35 \mathrm{~dB}$, (b) $L o G / 30 \mathrm{~dB}$, (c) $L o G / 24 \mathrm{~dB}$, (d) $R b / 35 \mathrm{~dB}$, (e) $R b / 30 \mathrm{~dB}$, (f) $R b / 24 \mathrm{~dB}$, (g) $C n / 35 \mathrm{~dB}$, (h) $C n / 30 \mathrm{~dB}$, (i) $C n / 24 \mathrm{~dB}$, (j) $F_{7} / 35 \mathrm{~dB}$, (k) $F_{7} / 30 \mathrm{~dB}$ and (l) $F_{7} / 24 \mathrm{~dB}$.

\subsection{Discussion}

From the experiments, above, the advantages of our methodology are listed following. First, our methodology use a fuzzy technique that is very robust to the noise because of its uncertainty characteristic [23]. In addition, in our proposal the membership functions are designed from a global estimation of statistics parameters on the image in contrast with the $F z$ approach [22]. In fact, the input membership functions are defined from both the region of uncertainty controlled by $\lambda_{1}$ and $\lambda_{2}$ and its shape characterized using the FCM algorithm. It allows less variability with respect to threshold. In addition, this method allows to choose the $\delta$ value that controls the mask definition.

To validate the latter, we chose a straightforward protocol experiment to determine the suitable set of parameters in our edge detection method. We adopted the F- 

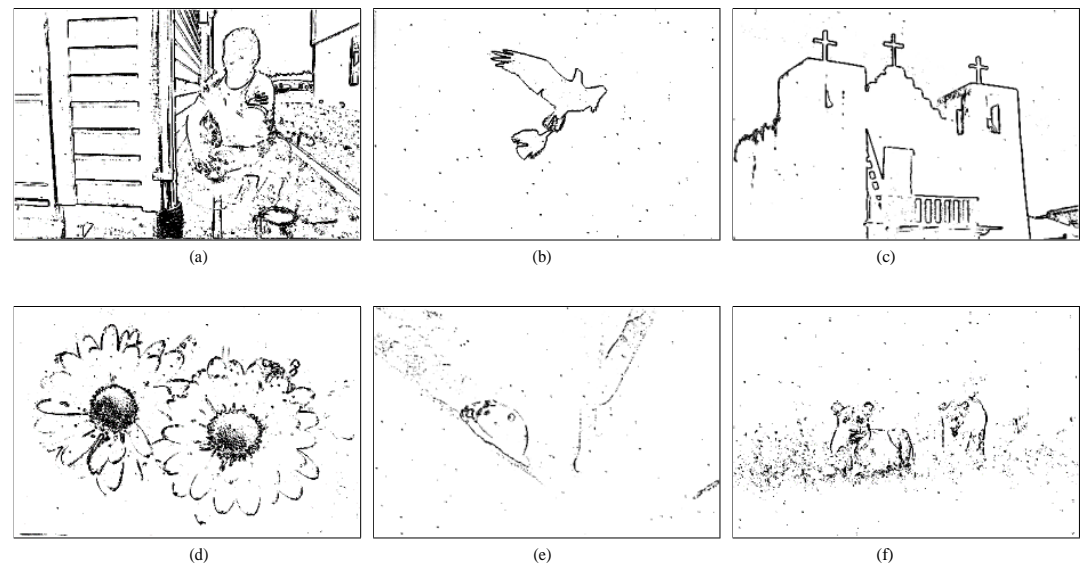

Figure 15

The edge maps resulting from the $F_{7}$ method applied to the $24 \mathrm{~dB}$ noisy images using a fixed threshold. IDs: (a) 23080, (b) 135069, (c) 24063, (d) 124084, (e) 35058 and (f) 105053.

measure metric to test sensibility and precision of the method. We also used it to confirm the adoption of a fixed threshold, handling the threshold choosing problem [33].

From the results, in both cases with and without noise, our methodology overcomes the traditional methods and compete with the fuzzy method of [22]. In particular, our method performs better in presence of noise and is also robust regardless of the threshold. In that way, we can argue the choosing of a fixed value. In addition, the performance of our method is invariant to different noise levels.

On the other hand, our methodology presents some limitations. For low contrast images our approach decreases its performance. However, it can be improved by changing the definition of the mask. Also, we noticed that the region of uncertainty has direct impact on the performance of the edge detection method. In this work, it has not been detected automatically.

\section{Conclusions}

In this work, we have proposed a fuzzy ensemble based method for edge detection. It includes a FCM strategy to define the input membership functions of the fuzzy inference system from a global estimation of statistics parameters on the image, and the characterization of the region of uncertainty.

We tested the performance of the method using a public database and we compared it with classical methods and the fuzzy based method presented in [22]. In order to find a suitable set of parameters in our method we tested it with different instances. The F-measure was used to evaluate the performance of the edge detectors. We also measured the standard deviation for the F-measure to confirm the invariability of our method with respect to the threshold in comparison to classical methods.

The method was also tested with three levels of salt \& pepper noise. Results val- 
idated that fuzzy ensemble based method overcomes the traditional methods and compete with the fuzzy method of [22]. In general, the metric values obtained from our proposal in the highest noise condition were better than the other fuzzy approach.

The advantages of our methodology include: the design of membership functions from a global estimation of statistics parameters on the image using FCM for shape characterization, the less variability with respect to threshold and the robustness to noise.

In comparison to the fuzzy method of [22], our methodology competes and extends its fixed parameters (window size and shape of membership functions) by including some tuning parameters. In addition, our approach allows the use of a constant threshold. Furthermore, the current work has some advantages with respect to other image fuzzy enhancement methods as [20] like the definition of the membership functions are not preset, an optimization algorithm is not required for threshold selection and an extensive quantitative analysis to compare to other methods was performed.

For future work we are considering to define another kind of mask to handle low contrast images and to design an automatic strategy to estimate the region of uncertainty. Also, other strategies to add high order information (e.g. texture features) to the inputs of the fuzzy system could be used to improve the performance of the edge detection method.

\section{References}

[1] Mathew SP, Balas VE, Zachariah KP, Samuel P. A content-based image retrieval system based on polar raster edge sampling signature. Acta Polytechnica Hungarica. 2014;11(3):25-36

[2] Mathew SP, Balas VE, Zachariah KP. A content-based image retrieval system based on convex hull geometry. Acta Polytechnica Hungarica. 2015;12(1):103-116

[3] Torre V, Poggio TA. On Edge Detection. IEEE Transactions on Pattern Analysis and Machine Intelligence. 1986;PAMI-8(2):147-163

[4] Arbelaez P, Maire M, Fowlkes C, Malik J. Contour Detection and Hierarchical Image Segmentation. IEEE Trans Pattern Anal Mach Intell. 2011;33(5):898-916

[5] Jin-Yu Z, Yan C, Xian-Xiang H. Edge detection of images based on improved Sobel operator and genetic algorithms. In: 2009 International Conference on Image Analysis and Signal Processing; 2009. pp. 31-35

[6] Rosenfeld A. The Max Roberts Operator is a Hueckel-Type Edge Detector. IEEE Transactions on Pattern Analysis and Machine Intelligence. 1981;PAMI-3(1):101-103 
[7] Yang L, Wu X, Zhao D, Li H, Zhai J. An improved Prewitt algorithm for edge detection based on noised image. In: Image and Signal Processing (CISP), 2011 4th International Congress on. Vol. 3; 2011. pp. 1197-1200

[8] Ulupinar F, Medioni G. Refining edges detected by a LoG operator. Computer Vision, Graphics, and Image Processing. 1990;51(3):275-298

[9] Canny J. A Computational Approach to Edge Detection. IEEE Transactions on Pattern Analysis and Machine Intelligence. 1986;PAMI-8(6):679-698

[10] Genming C, Baozong YAA. A new edge detector with thinning and noise resisting abilities. Journal of Electronics (China). 1989;6(4):314-319

[11] Wang X, Zhang X, Gao R. An adaptive edge detection algorithm based on gray-scale morphology. In: Measurement, Information and Control (ICMIC), 2013 International Conference on. Vol. 02; 2013. pp. 1251-1254

[12] Jiang J, Chuang C, Lu Y, Fahn C. Mathematical-morphology-based edge detectors for detection of thin edges in low-contrast regions. IET Image Processing. 2007;1(3):269-277

[13] Guo X, Xu Z, Lu Y, Pang Y. An Adaptive Edge Detector Using Soft Mathematical Morphology. In: The Fifth International Conference on Computer and Information Technology (CIT’05); 2005. pp. 608-607

[14] Krishnamurthy S, Iyengar SS, Holyer RJ, Lybanon M. Histogram-based morphological edge detector. IEEE Transactions on Geoscience and Remote Sensing. 1994;32(4):759-767

[15] Singh H, Kaur G, Gupta N. Robust edge detector using back propagation neural network with multi-thresholding. In: Computational Intelligence and Computing Research (ICCIC), 2014 IEEE International Conference on; 2014. pp. 1-6

[16] Li N, Huo H, m Zhao Y, Chen X, Fang T. A Spatial Clustering Method With Edge Weighting for Image Segmentation. IEEE Geoscience and Remote Sensing Letters. 2013;10(5):1124-1128

[17] Boskovitz V, Guterman H. An adaptive neuro-fuzzy system for automatic image segmentation and edge detection. IEEE Transactions on Fuzzy Systems. 2002;10(2):247-262

[18] Khunteta A, Ghosh D. Edge detection via fuzzy rule-based edge strength estimation and optimal threshold selection using PSO. In: 2013 IEEE $8^{\text {th }}$ International Conference on Industrial and Information Systems; 2013. pp. $560-565$

[19] Talai Z, Talai A. A fast edge detection using fuzzy rules. In: Communications, Computing and Control Applications (CCCA), 2011 International Conference on; 2011. pp. 1-5

[20] Zhang D, Zhan B, Yang G, Hu X. An improved edge detection algorithm 
based on image fuzzy enhancement. In: $20094^{\text {th }}$ IEEE Conference on Industrial Electronics and Applications; 2009. pp. 2412-2415

[21] Setarehdan SK, Soraghan JJ. Automatic cardiac LV boundary detection and tracking using hybrid fuzzy temporal and fuzzy multiscale edge detection. IEEE Transactions on Biomedical Engineering. 1999;46(11):1364-1378

[22] Haq I, Anwar S, Shah K, Khan MT, Shah SA. Fuzzy Logic Based Edge Detection in Smooth and Noisy Clinical Images. PLoS ONE. 2015;10(9):117

[23] Guillaume S. Designing Fuzzy Inference Systems from Data: An Interpretability-Oriented Review. IEEE Transactions on Fuzzy Systems. 2001;9(3):426-443

[24] Kim S, Lee M, Lee J. A Study of Fuzzy Membership Functions for Dependence Decision-Making in Security Robot System. Neural Computing and Applications. 2015; pp. 1-10

[25] Shtovba SD. Fuzzy model Tuning Based on a Training Set With Fuzzy Model Output Values. Cybernetics and Systems Analysis. 2007;43(3):334-340

[26] Iancu I. 16. In: Dadios E, editor. A Mamdani Type Fuzzy Logic Controller. InTech; 2012. pp. 325-350

[27] Bezdek JC. Pattern Recognition With Fuzzy Ocjective Function Algorithms. Norwell, MA: Kluwer Academic Publishers; 1981

[28] Martin D, Fowlkes C, Tal D, Malik J. A Database of Human Segmented Natural Images and its Application to Evaluating Segmentation Algorithms and Measuring Ecological Statistics. In: Proc. $8^{\text {th }}$ Int'l Conf. Computer Vision. Vol. 2; 2001.pp. 416-423

[29] Martin D, Fowlkes C, Tal D, Malik J. The Berkeley Segmentation Dataset and Benchmark; 2007. Online. Available from: http://www.eecs.berkeley. edu/Research/Projects/CS/vision/bsds/

[30] Goldberg A. Andrew Goldberg's Network Optimization Library; 1995. Online. Available from: http://www.avglab.com/andrew/soft.html

[31] Martin DR, Fowlkes CC, Malik J. Learning to detect natural image boundaries using local brightness, color, and texture cues. IEEE Transactions on Pattern Analysis and Machine Intelligence. 2004;26(5):530-549

[32] Rijsbergen CJv. Information Retrieval. $2^{\text {nd }}$ ed. Newton, MA, USA: Butterworth-Heinemann; 1979. Available from: http://www.dcs.gla. ac.uk/Keith/pdf/Chapter7.pdf

[33] Kim DS, Lee WH, Kweon IS. Automatic edge detection using 3x3 ideal binary pixel patterns and fuzzy-based edge thresholding. Pattern Recognition Letters. 2004;25(1):101-106 